\title{
Lower Bounds for Oblivious Transfer Reductions
}

\author{
Yevgeniy Dodis $^{1}$ and Silvio Micali ${ }^{2}$ \\ 1 Laboratory for Computer Science, Massachusetts Institute of Technology, USA \\ yevgen@theory.lcs.mit.edu \\ ${ }^{2}$ Laboratory for Computer Science, Massachusetts Institute of Technology, USA \\ silvio@tiac.net
}

\begin{abstract}
We prove the first general and non-trivial lower bound for the number of times a 1-out-of-n Oblivious Transfer of strings of length $\ell$ should be invoked so as to obtain, by an information-theoretically secure reduction, a 1-out-of-N Oblivious Transfer of strings of length $L$. Our bound is tight in many significant cases.

We also prove the first non-trivial lower bound for the number of random bits needed to implement such a reduction whenever the receiver sends no messages to the sender. This bound is also tight in many significant cases.
\end{abstract}

\section{Introduction}

The OBlivious Transfer. The Oblivious Transfer (OT) is a fundamental primitive in secure protocol design, which has been defined in many different ways and contexts (e.g. [17, [10], 99]) and has found enormously many applications (e.g. [2], [17, 9], 13], 7], 16], [1, 14], 11]).

The OT is a protocol typically involving two players, the sender and the receiver, and several parameters. In the most used form, the $\left(\begin{array}{c}N \\ 1\end{array}\right)-\mathrm{OT} \mathrm{T}_{2}^{L}$, the sender has $N$ binary secrets of length $L$, and the receiver gets exactly one of these strings, the one he chooses, but no information about any other secret (even if he cheats), while the sender (even if she cheats) gets no information about the secret learned by the receiver.

Also important is the notion of a weak Oblivious Transfer, a relaxation of the traditional OT. The only difference in a weak $\left(\begin{array}{c}N \\ 1\end{array}\right)-\mathrm{OT}_{2}^{L}$ is that a cheating receiver is allowed to obtain partial information about several secrets, but at most $L$ bits of information overall.

ReDuCtions Between DifFEREnT OTs. Protocol reductions facilitate protocol design because they enable one to take advantage of implementing cryptographically only a few, carefully chosen, primitives. Information-theoretic reductions are even more attractive, because they guarantee that the security of a complex construction automatically coincides with that of the chosen primitive, once the latter is implemented cryptographically. 
But to be really useful, reductions must be efficient. In particular, because even the best cryptographic implementation of a chosen primitive may be expensive to run, it is crucial that reductions call such primitives as few times as possible.

Because of the importance of OT, numerous reductions from "more complex" to "simpler" OT appear in the literature (e.g. [5, 8], 3, 6]). Particular attention has been devoted to reducing $\left(\begin{array}{c}N \\ 1\end{array}\right)-\mathrm{OT}_{2}^{L}$ to $\left(\begin{array}{c}n \\ 1\end{array}\right)-\mathrm{OT}_{2}^{\ell}$, where $N \geq n$ and $L \geq \ell$, both in the weak and in the strong case. Typically, these reductions are information-theoretically secure if the simpler OT is assumed to be so secure.

An important class of OT reductions are the ones in which the receiver sends no messages to the sender. Such reductions are called natural, both because all known OT reductions are of this type (e.g. 5], 6], 3]), and because they immediately imply that the sender gets no information about the receiver's index.

So far, researchers have been focusing on improving the upper bounds of these reductions, that is, the number of times one calls $\left(\begin{array}{l}n \\ 1\end{array}\right)-\mathrm{OT}_{2}^{\ell}$ in order to construct $\left(\begin{array}{c}N \\ 1\end{array}\right)-\mathrm{OT}_{2}^{L}$. However, little is known about the corresponding lower bounds. Indeed,

What is the minimum number of times that the given $\left(\begin{array}{l}n \\ 1\end{array}\right)-O T_{2}^{\ell}$ must be invoked so as to obtain the desired $\left(\begin{array}{c}N \\ 1\end{array}\right)-O T_{2}^{L}$ ?

Lower bounds were previously addressed in the context of very specific reduction techniques, and for very specific OTs. For instance, in [5] simple lower bounds are derived for reductions of $\left(\begin{array}{l}2 \\ 1\end{array}\right)-\mathrm{OT}_{2}^{L}$ to $\left(\begin{array}{l}2 \\ 1\end{array}\right)-\mathrm{OT}_{2}^{1}$ that use zigzag functions.

Another natural resource of a reduction of $\left(\begin{array}{c}N \\ 1\end{array}\right)-\mathrm{OT}_{2}^{L}$ to $\left(\begin{array}{c}n \\ 1\end{array}\right)-\mathrm{OT}_{2}^{\ell}$ is the amount of needed randomness. That is, an OT protocol is necessary probabilistic, but

What is the minimum number of random bits needed in a informationtheoretically secure reduction of $\left(\begin{array}{c}N \\ 1\end{array}\right)-O T_{2}^{L}$ to $\left(\begin{array}{c}n \\ 1\end{array}\right)-O T_{2}^{\ell}$ ?

To the best of our knowledge, no significant results have ever been obtained about this crucial aspect.

OUR RESULTS. In this paper we provide the first general lower bounds for such information-theoretic OT reductions, and prove that these bounds are tight in significant cases. Namely, we prove that

- In any information-theoretically secure reduction of (even weak!) $\left(\begin{array}{c}N \\ 1\end{array}\right)$-OT $\mathrm{OT}_{2}^{L}$ to $\left(\begin{array}{c}n \\ 1\end{array}\right)-\mathrm{OT}_{2}^{\ell}$, the latter protocol must be invoked at least $\frac{L}{\ell} \cdot \frac{N-1}{n-1}$ times.

- The lower bound is tight for weak $\left(\begin{array}{c}N \\ 1\end{array}\right)-\mathrm{OT}_{2}^{L}$.

- The lower bound is tight for ("strong") $\left(\begin{array}{c}N \\ 1\end{array}\right)-\mathrm{OT}_{2}^{L}$ when $L=\ell$.

We also prove the first general lower bound for the amount of randomness needed in a natural OT reduction. Namely,

- In any natural reduction of (even weak) $\left(\begin{array}{c}N \\ 1\end{array}\right)-\mathrm{OT}_{2}^{L}$ to $\left(\begin{array}{c}n \\ 1\end{array}\right)-\mathrm{OT}_{2}^{\ell}$, the sender must flip at least $\frac{L(N-n)}{n-1}$ coins. 
- The lower bound is tight for weak $\left(\begin{array}{c}N \\ 1\end{array}\right)-\mathrm{OT}_{2}^{L}$.

- The lower bound is tight for ("strong") $\left(\begin{array}{c}N \\ 1\end{array}\right)-\mathrm{OT}_{2}^{L}$ when $L=\ell$.

We note that, in a natural reduction, the amount of randomness used by the sender necessarily coincides with the total amount of randomness needed by both parties.

We point out the interesting special case when $n=2$ and $\ell=1$, i.e. reducing $\left(\begin{array}{c}N \\ 1\end{array}\right)-\mathrm{OT}_{2}^{L}$ to $\left(\begin{array}{l}2 \\ 1\end{array}\right)-\mathrm{OT}_{2}$, the simplest possible 1-out-2 Oblivious Transfer. We obtain that we need at least $L(N-1)$ invocations of $\left(\begin{array}{l}2 \\ 1\end{array}\right)-\mathrm{OT}_{2}$ and, for a natural OT reduction, at least $L(N-2)$ random bits.

LOWER BOUNDS VIA INFORMATION THEORY. No general lower bound for OT reduction would be provable without very precisely and generally defining what such a reduction is. Fortunately, one such definition was successfully given by Brassard, Crépeau, and Sántha [5] based on information theory, and in particular the notion of mutual information. This framework is very useful since it allows one to define precisely such intuitive (but hard to capture formally) notions as "learn at most $k$ bits of information" or "learn no information other than ...".

We point out, however, that information theory is much more useful than merely defining the problem. Indeed, we shall demonstrate that its powerful machinery is essential in solving our problem, for example, in proving our $\frac{L}{\ell} \cdot \frac{N-1}{n-1}$ lower bound on the number of invocations. Only the trivial bound of $\frac{L}{\ell}$ appears to be provable without information theory. But getting the additional $\frac{N-1}{n-1}$ factor in the lower bound (which is essential when $L=\ell$ ) requires explicit or implicit use of information theory.

We believe and hope that information theory will prove useful for other types of lower bounds in protocol problems.

\section{Preliminaries}

\subsection{Information Theory Background}

Let $X, Y, Z$ by random variables over domains $\mathcal{X}, \mathcal{Y}, \mathcal{Z}$. Let us denote by $P_{X}(x)$, $P_{X \mid Z}(x \mid z), P_{X, Y}(x, y)$ the probability distribution of $X$, conditional probability distribution of $X$ given $Z$, and joint distribution of $X$ and $Y$ respectively.

\section{Definition 1.}

- The entropy $\mathbf{H}(X)=-\sum_{x} P_{X}(x) \log _{2} P_{X}(x)$.

The entropy of a random variable $X$ tells how many truly random bits one can extract from $X$, i.e. how much "uncertainty" is in $X$.

- The conditional entropy $\mathbf{H}(X \mid Z)$ is the average over $z$ of the entropy of the variable $X_{z}$ distributed according to $P_{X \mid Z}(x \mid z)$ (denoted $\mathbf{H}(X \mid Z=z)$ ), i.e.

$$
\mathbf{H}(X \mid Z)=\sum_{z} P_{Z}(z) \mathbf{H}(X \mid Z=z)=-\sum_{z} P_{Z}(z) \sum_{x} P_{X \mid Z}(x \mid z) \log _{2} P_{X \mid Z}(x \mid z)
$$

$\mathbf{H}(X \mid Z)$ measures how much uncertainty $X$ still has when one knows $Z$. 
- The joint entropy of $X$ and $Y$ is the entropy of the joint variable $(X, Y)$, i.e.

$$
\mathbf{H}(X, Y)=-\sum_{x, y} P_{X, Y}(x, y) \log _{2} P_{X, Y}(x, y)
$$

- The mutual information between $X$ and $Y$ is $\mathbf{I}(X ; Y)=\mathbf{H}(X)-\mathbf{H}(X \mid Y)$.

- The mutual information between $X$ and $Y$ given $Z$ is $\mathbf{I}(X ; Y \mid Z)=\mathbf{H}(X \mid Z)$ $\mathbf{H}(X \mid Y, Z)$.

The mutual information between $X$ and $Y$ (given $Z$ ) tells how much common information is between $X$ and $Y$ (given $Z$ ), i.e. by how much the uncertainty of $X$ (given $Z$ ) decreases after one learns $Y$.

The following easily verified lemma summarizes some of the properties we will need.

\section{Lemma 2.}

1. $\mathbf{H}(X, Y)=\mathbf{H}(X)+\mathbf{H}(Y \mid X)=\mathbf{H}(Y)+\mathbf{H}(X \mid Y)$.

2. $\mathbf{I}(X ; Y)=\mathbf{I}(Y ; X)=\mathbf{H}(Y)-\mathbf{H}(Y \mid X)=\mathbf{H}(X)-\mathbf{H}(X \mid Y)=\mathbf{H}(X)+\mathbf{H}(Y)-$ $\mathbf{H}(X, Y)$.

3. $\mathbf{I}(X, Z ; Y)=\mathbf{I}(X ; Y)+\mathbf{I}(Z ; Y \mid X)$.

4. $\mathbf{H}(X \mid Y)=0$ iff $X$ is a deterministic function of $Y$.

5. $\mathbf{H}(X \mid Y) \leq \mathbf{H}(X)$ with equality iff $X$ and $Y$ are independent.

(Thus, $\mathbf{I}(X ; Y) \geq 0$ with equality iff $X$ and $Y$ are independent.)

6. $\mathbf{I}(X ; Y) \leq \mathbf{H}(X) \leq \log _{2}|\mathcal{X}|$.

7. $\mathbf{I}(X ; Y) \leq \mathbf{I}(X ; Y \mid Z)+\mathbf{H}(Z)$.

8. $\mathbf{H}\left(U_{n}\right)=n$, where $U_{n}$ is the uniform distribution over $n$-bit strings.

\subsection{Information-Theoretically Secure OT Reductions}

Assuming some familiarity with the notions of an interactive Turing machine (ITM) 12, let us semi-formally define (1) protocols with an ideal $\left(\begin{array}{l}n \\ 1\end{array}\right)-\mathrm{OT}_{2}^{\ell}$ and then (2) information-theoretically secure reduction of $\left(\begin{array}{c}N \\ 1\end{array}\right)-\mathrm{OT}_{2}^{L}$ to $\left(\begin{array}{c}n \\ 1\end{array}\right)-\mathrm{OT}_{2}^{\ell}$.

Despite the difference in presentation, the following definition is a simplification of that of [5]. (For instance, we simplify it by ignoring the additional condition of awareness that is not going to affect our lower bound in any way.)

Protocols with ideal $\left(\begin{array}{l}n \\ 1\end{array}\right)$-OT 2 . Let us denote by a $n$-sender a probabilistic ITM having $n$ special registers, and by a $n$-receiver is probabilistic ITM having a single special register. Let $A$ be a $n$-sender and $B$ a $n$-receiver. We say that $(A, B)$ is a protocol with ideal $\left(\begin{array}{l}n \\ 1\end{array}\right)-O T_{2}^{\ell}$ if, letting $a$ be a private input for $A$ and $b$ be a private input for $B$, the computation of $(A, B)$ proceeds as that of pair of ITMs, except that it consists of three (rather than the usual two) types of rounds: sender-rounds, receiver-rounds and OT-rounds, where by convention the first round always is a sender-round and the last is a receiver-round. In a sender-round, only $A$ is active, and it sends a message to $B$ (that will become an input to $B$ at the start of the next receiver-round). In a receiver-round, only $B$ is active and, except for the last round, it sends a message to $A$ (this message will become an input to $A$ at the start of the next sender-round). In an OT round, 
(1) $A$ places for each $j \in[1, n]$ an $\ell$-bit string $\sigma_{j}$ in its $j$ th special register, and

(2) $B$ places an integer $i \in[1, n]$ in its special register, and

(3) $\sigma_{i}$ will become a distinguished input to $B$ at the start of the next receiverround. $A$ will obtain no information about $i$.

At the end of any execution of $(A, B), B$ computes a distinguished string called B's output.

Messages And Views. Let $(A, B)$ be a protocol with ideal $\left(\begin{array}{l}n \\ 1\end{array}\right)$-OT $\mathrm{OT}_{2}^{\ell}$. Then, in an execution of $(A, B)$, we refer to the messages that $A$ sends in a sender-round as $A$ 's ordinary messages, and to the strings that $A$ writes in its special registers in an OT-round as A's potential OT messages. For each OT-round, only one of the $n$ potential messages will be received by $B$, and we shall refer to all such received messages as $B$ 's actual OT messages. Recalling that both $A$ and $B$ are probabilistic, in a random execution of $(A, B)$ where the private input of $A$ is $a$ and the private input of $B$ is $b$, let us denote by $\operatorname{VIEW}_{A}[A(a), B(b)]$ the random variable consisting of

(1) $a,(2) A$ 's coin tosses, and (3) the ordinary messages received by $A$; and let us denote by $\operatorname{VIEW}_{B}[A(a), B(b)]$ the random variable consisting of

(1) $b,(2) B$ 's coin tosses, and (3) all messages (both the ordinary and the actual OT ones) received by $B$.

Reduction of $\left(\begin{array}{c}N \\ 1\end{array}\right)-\mathrm{OT}_{2}^{L}$ то $\left(\begin{array}{c}n \\ 1\end{array}\right)-\mathrm{OT}_{2}^{\ell}$. Denote by $\mathcal{W}$ the set of all $N$-long sequences of $L$-bit stings and, given $w \in \mathcal{W}$, let $w_{i}$ be the $i^{\text {th }}$ string of $w$. Denote by $W$ the random variable that selects an element of $\mathcal{W}$ with uniform probability; by $I$ the random variable selecting an integer in $[1, N]$ with uniform probability; and let $A$ be an $n$-sender and $B$ be an $n$-receiver. We say that $(A, B)$ is an information-theoretically secure reduction of $\left(\begin{array}{c}N \\ 1\end{array}\right)-\mathrm{OT}_{2}^{L}$ to $\left(\begin{array}{l}n \\ 1\end{array}\right)-\mathrm{OT}_{2}^{\ell}$ if the following three properties are satisfied:

(P1) (Correctness) $\forall w \in \mathcal{W}$ and $\forall i \in[1, N]$, and $\forall$ execution of $(A, B)$ where $A$ 's private input is $w$ and $B$ 's private input is $i$,

$$
B \text { 's output is } w_{i} \text {; }
$$

(P2) (Receiver Privacy) $\forall$ sender $A^{\prime}$ and $\forall$ string $a^{\prime}$,

$$
\mathbf{I}\left(\mathrm{VIEW}_{A^{\prime}}\left[A^{\prime}\left(a^{\prime}\right), B(I)\right] ; I\right)=0 ;
$$

(P3) (Sender Privacy) $\forall$ receiver $B^{\prime}$ and string $b^{\prime}, \exists$ a random variable $\tilde{I} \in[1, N]$ independent of $W$ s.t.

$$
\mathbf{I}\left(W ; \operatorname{VIEW}_{B^{\prime}}\left[A(W), B^{\prime}\left(b^{\prime}\right)\right] \mid W_{\tilde{I}}\right)=0 .
$$

In the context of a reduction of $\left(\begin{array}{c}N \\ 1\end{array}\right)-\mathrm{OT}_{2}^{L}$ to $\left(\begin{array}{c}n \\ 1\end{array}\right)-\mathrm{OT}_{2}^{\ell}$, we shall sometimes say that we are given $\left(\begin{array}{l}n \\ 1\end{array}\right)-\mathrm{OT}_{2}^{\ell}$ as a black-box. 
The Correctness Property states that when $A$ and $B$ are honest, $B$ will always obtain the string he wants. The Receiver Privacy Property states that no malicious sender $A^{\prime}$ can learn any information about the index of the honest receiver $B$. Finally, the Sender Privacy Property states that a malicious receiver $B^{\prime}$ can learn information about at most one of $N$ strings of the sender $A$. Moreover, the index $\tilde{I}$ of this single string cannot depend on $W$ (e.g. we don't want $B^{\prime}$ to learn the first string in $W$ that starts with 10). In other words, both $A$ and $B$ do not gain anything by not following the protocol.

REDUCTION OF WEAK $\left(\begin{array}{c}N \\ 1\end{array}\right)-\mathrm{OT}_{2}^{L}$ TO $\left(\begin{array}{c}n \\ 1\end{array}\right)-\mathrm{OT}_{2}^{\ell}$. We call $(A, B)$ an informationtheoretically secure reduction of weak $\left(\begin{array}{c}N \\ 1\end{array}\right)-\mathrm{OT}_{2}^{L}$ to $\left(\begin{array}{c}n \\ 1\end{array}\right)-\mathrm{OT}_{2}^{\ell}$ if all the properties of the reduction of $\left(\begin{array}{c}N \\ 1\end{array}\right)-\mathrm{OT}_{2}^{L}$ to $\left(\begin{array}{c}n \\ 1\end{array}\right)-\mathrm{OT}_{2}^{\ell}$ hold except (Sender Privacy) is relaxed to the following:

$\left(\mathrm{P} 3^{\prime}\right)$ (Weak Sender Privacy) $\forall$ receiver $B^{\prime}$ and string $b^{\prime}$

$$
\mathbf{I}\left(W ; \operatorname{VIEW}_{B^{\prime}}\left[A(W), B^{\prime}\left(b^{\prime}\right)\right]\right) \leq L
$$

This property says that we allow a malicious receiver $B^{\prime}$ to obtain partial information about possibly several strings, provided he learns no more than $L$ bits of information overall. To emphasize the difference, we will sometimes refer to the (regular) reduction between $\left(\begin{array}{c}N \\ 1\end{array}\right)-\mathrm{OT}_{2}^{L}$ and $\left(\begin{array}{c}n \\ 1\end{array}\right)-\mathrm{OT}_{2}^{\ell}$ as reducing strong $\left(\begin{array}{c}N \\ 1\end{array}\right)$-OT $\mathrm{OT}_{2}^{L}$ to $\left(\begin{array}{l}n \\ 1\end{array}\right)-\mathrm{OT}_{2}^{\ell}$. To justify this terminology, we show

Lemma 3. If $(A, B)$ is a reduction of (strong) $\left(\begin{array}{c}N \\ 1\end{array}\right)-O T_{2}^{L}$ to $\left(\begin{array}{c}n \\ 1\end{array}\right)-O T_{2}^{\ell}$, then it is a reduction of weak $\left(\begin{array}{c}N \\ 1\end{array}\right)-O T_{2}^{L}$ to $\left(\begin{array}{c}n \\ 1\end{array}\right)-O T_{2}^{\ell}$.

Proof. By Lemma 2 (equations 7 and 6),

$$
\begin{aligned}
\mathbf{I}\left(W ; \operatorname{VIEW}_{B^{\prime}}\left[A(W), B^{\prime}\left(b^{\prime}\right)\right]\right) & \leq \mathbf{I}\left(W ; \operatorname{VIEW}_{B^{\prime}}\left[A(W), B^{\prime}\left(b^{\prime}\right)\right] \mid W_{\tilde{I}}\right)+\mathbf{H}\left(W_{\tilde{I}}\right) \\
& =\mathbf{H}\left(W_{\tilde{I}}\right) \leq\left|W_{\tilde{I}}\right|=L
\end{aligned}
$$

\section{Lower Bounds}

To simplify our notation, we do not worry about "floors" and "ceilings" in the rest of the paper, assuming that $(N-1)$ is divisible by $(n-1)$ and that $L$ is divisible by $\ell$ (handling the the general case presents no significant difficulties). We will also refer to the sender as Alice and to the receiver as Bob.

Let $\alpha$ be the number of OT-rounds (invocations of $\left(\begin{array}{l}n \\ 1\end{array}\right)-\mathrm{OT}_{2}^{\ell}$ ) needed to reduce (weak) $\left(\begin{array}{c}N \\ 1\end{array}\right)-\mathrm{OT}_{2}^{L}$ to $\left(\begin{array}{c}n \\ 1\end{array}\right)-\mathrm{OT}_{2}^{\ell}$. Since we concentrate on the worst possible number of OT-rounds, we can assume w.l.o.g. that $\alpha$ is a fixed number and that the sender and receiver always perform exactly $\alpha$ OT-steps. We start with a sharp lower bound on $\alpha$. 


\subsection{Lower Bound on the Number of Invocations of $\left(\begin{array}{l}n \\ 1\end{array}\right)-\mathrm{OT}_{2}^{\ell}$}

Theorem 4. Any information-theoretically secure reduction of weak $1\left(\begin{array}{c}N \\ 1\end{array}\right)-O T_{2}^{L}$ to $\left(\begin{array}{l}n \\ 1\end{array}\right)-O T_{2}^{\ell}$ must have

$$
\alpha \geq \frac{L}{\ell} \cdot \frac{N-1}{n-1}
$$

Proof. Let us first give the informal intuition behind the proof. We know by the (weak) sender privacy condition that Bob can learn at most $L$ (out of total $N L$ ) bits of information about $W$. However, if in each of the OT rounds Bob was somehow able to obtain all $n$ strings that Alice put as her local inputs to this OT round (rather than getting only one of them), Bob should be able to learn all ( $N L$ bits) of $W$. Indeed, if Bob could not cannot learn some $W_{i}$ with certainty, Alice will know that Bob's index is not $i$ (if it was $i$, honest Bob should be able to get $W_{i}$ with probability 1 by the correctness property). But this would contradict the receiver privacy condition as Alice learns some information about Bob's index. Hence, $\alpha n \ell-n \ell=\alpha \ell(n-1)$ bits that Bob did not get from the OT rounds, "contain information" about the remaining at least $N L-L=L(N-1)$ bits of $W$ that Bob did not learn. The bound follows.

Let us now turn this intuition into a formal proof. Let $P, P=($ Alice, Bob), be an information-theoretically secure reduction of $\left(\begin{array}{c}N \\ 1\end{array}\right)-\mathrm{OT}_{2}^{L}$ to $\left(\begin{array}{l}n \\ 1\end{array}\right)-\mathrm{OT}_{2}^{\ell}$ that uses $\alpha$ invocations to $\left(\begin{array}{l}n \\ 1\end{array}\right)-\mathrm{OT}_{2}^{\ell}$. First, we need the following simple lemma.

Local Lemma: $\forall$ input $w=w_{1}, \ldots, w_{N}, \forall$ random tape $R_{A}$ for Alice, $\forall$ distinct $i, i^{\prime} \in[1, N]$ and $\forall$ random tape tape $R_{B}^{\prime}$ for Bob, there exists a tape $R_{B}$ for Bob such that the sequence of messages, $M$, received by $\operatorname{Alice}\left(w, R_{A}\right)$ from $\operatorname{Bob}\left(i^{\prime}, R_{B}^{\prime}\right)$ coincides with the sequence of messages that $\operatorname{Alice}\left(w, R_{A}\right)$ receives from $\operatorname{Bob}\left(i, R_{B}\right)$.

Proof: Assume that $R_{B}$ does not exist. Then, executing with $\operatorname{Bob}\left(i^{\prime}, R_{B}^{\prime}\right)$, we get that $\operatorname{Alice}\left(w, R_{A}\right)$ will determine for sure that Bob's index is not $i$. Thus, when Bob's index is $i^{\prime}$, with non-zero probability over Bob's random string, Alice $\left(w, R_{A}\right)$ would obtain information about Bob's index (that it is not $i$ ), contradicting the receiver privacy condition.

To derive our lower bound for $\alpha$, we define the following two notions: that of a special execution of $P$ and that of a pseudo-execution of $P$.

Special execution. A special execution of $P$ is an execution of $P$ in which Alice's input is a sequence of $N$ randomly selected strings of length $L$, Alice's tape consists of randomly and independently selected bits, Bob's index is 1, and Bob's tape is the all-zero string, $\mathbf{0}$. In other words, we fix the behavior of Bob by fixing his index and the random string. With respect to a special execution of $P$, define the following random variables:

- $W$ - Alice's $N L$-bit strings, $W=W_{1}, \ldots, W_{N}$;

$-R$ - Alice's random tape;

\footnotetext{
${ }^{1}$ Since we are proving a lower bound, it clearly applies to (strong) $\left(\begin{array}{c}N \\ 1\end{array}\right)$-OT $\mathrm{OT}_{2}^{L}$ as well.
} 
- $M_{s}$ - the ordinary messages sent by sender Alice;

- $M_{r}$ — the ordinary messages sent by receiver Bob;

- $V$ - Alice's potential messages (an $\alpha n \ell$-bit string, that is, for each of the $\alpha$ invocations of $\left(\begin{array}{l}n \\ 1\end{array}\right)$-OT $T_{2}^{\ell}$, the $n \ell$-bit strings that are Alice's local inputs in the invocation).

- $V_{r}$ - the actual messages received by Bob in the OT-rounds, (an $\alpha \ell$-bit string, that is, for each of the $\alpha$ invocations of $\left(\begin{array}{l}n \\ 1\end{array}\right)-\mathrm{OT}_{2}^{\ell}$, the $\ell$-bit string that Bob received depending on his local index during that invocation).

Pseudo-execution. Let $\bar{M}_{s}$ be a sequence of messages, let $\bar{V}$ be a sequence of $\alpha$ sequences of $n$ strings of length $l$ each, let $\bar{i}$ be an index in $[1, N]$, and let $\bar{R}_{B}$ be a bit-sequence. A pseudo-execution of $P$ with inputs $\bar{M}_{s}, \bar{V}$, $\bar{i}$, and $\bar{R}_{B}$, denoted by $\bar{P}\left(\bar{M}_{s}, \bar{V}, \bar{i}, \bar{R}_{B}\right)$, is the process of running Bob with index $\bar{i}$ and coin tosses $\bar{R}_{B}$, letting the $k^{t h}$ message from the sender be the $k^{\text {th }}$ string of $\bar{M}_{s}$, and by letting the sender's input to the $j^{\text {th }}$ invocation of $\left(\begin{array}{l}n \\ 1\end{array}\right)$-OT $T_{2}^{\ell}$ to be the $j^{t h} n$-tuple of $l$-bit strings in $\bar{V}$. In other words, we pretend to be Alice and see what Bob will do in this situation on some particular index and random string.

Our lower bound for $\alpha$ immediately follows from the following two claims.

Local Claim 1: $\mathbf{I}\left(\left(V, M_{s}\right) ; W\right)=N L$.

Proof: By the definition of mutual information, we have

$$
\mathbf{I}\left(\left(V, M_{s}\right) ; W\right)=\mathbf{H}(W)-\mathbf{H}\left(W \mid\left(V, M_{s}\right)\right) .
$$

Because $W$ is randomly selected, $\mathbf{H}(W)=N L$. Therefore, to establish our claim we must prove that $\mathbf{H}\left(W \mid\left(V, M_{s}\right)\right)=0$. We do that by showing that $W$ is computable from $V$ and $M_{s}$ by means of the following algorithm.

1. Run $\bar{P}\left(V, M_{s}, 1, \mathbf{0}\right)$ and let $M_{r}$ be the resulting "ordinary messages sent by Bob".

(Comment: Bob's view and Bob's messages sent in this pseudo-execution are distributed exactly as in a special execution.)

2. For $i=1 \ldots N$ compute $W_{i}$ as follows:

- Find a string $R_{i}$ such that, when executing $\bar{P}\left(V, M_{s}, i, R_{i}\right)$, the sequence of messages sent by Bob equals $M_{r}$.

(Comment: The existence of at least one such $R_{i}$ follows from the Local Lemma with $i^{\prime}=1, R_{B}^{\prime}=\mathbf{0}, w=W$ and $R_{A}=R$. Further notice that, because $M_{r}, W$ and $R$ totally determine Alice's behavior, the messages and "potential" messages that $\operatorname{Alice}(W, R)$ sends to $B o b(1, \mathbf{0})$ and to $\operatorname{Bob}\left(i, R_{i}\right)$ are exactly $V$ and $M_{s}$ in both cases. Hence, any $R_{i}$ that produces $M_{r}$ in the pseudo-execution $\bar{P}\left(V, M_{s}, i, R_{i}\right)$, implies that $\operatorname{Alice}(W, R)$ would produce messages $M_{s}$ and "potential" messages $V$ when communicating with $\operatorname{Bob}\left(i, R_{i}\right)$.)

- Let $W_{i}$ be $B o b$ 's output in $\bar{P}\left(V, M_{s}, i, R_{i}\right)$.

(Comment: By the correctness property of our reduction, $B o b\left(i, R_{i}\right)$ would correctly output $W_{i}$ when talking to $\operatorname{Alice}(W, R)$. And as we noticed, Alice $(W, R)$ would produce $M_{s}$ and $V$ when communicating with 
$\operatorname{Bob}\left(i, R_{i}\right)$, so running pseudo-execution $\bar{P}\left(V, M_{s}, i, R_{i}\right)$ indeed makes Bob to produce the correct $\left.W_{i}\right)$.

Local Claim 2: $\mathbf{I}\left(\left(V, M_{s}\right) ; W\right) \leq L+\alpha \ell(n-1)$.

Proof: By Lemma 2 (equation 3), we have

$$
\mathbf{I}\left(\left(V, M_{s}\right) ; W\right)=\mathbf{I}\left(\left(V_{r}, M_{s}\right) ; W\right)+\mathbf{I}\left(\left(V \backslash V_{r}\right) ; W \mid\left(V_{r}, M_{s}\right)\right) .
$$

Now, because $P$ implements weak $\left(\begin{array}{c}N \\ 1\end{array}\right)-\mathrm{OT}_{2}^{L}$, and because $\left(V_{r}, M_{s}\right)$ consists of Bob's view in a (special) execution of $P$, we have by $\left(\mathrm{P} 3^{\prime}\right)$ that $\mathbf{I}\left(\left(V_{r}, M_{s}\right) ; W\right) \leq$ L. Also, by Lemma 2 (equations 5 and 6 ),

$$
\mathbf{I}\left(\left(V \backslash V_{r}\right) ; W \mid\left(V_{r}, M_{s}\right)\right) \leq\left|V \backslash V_{r}\right|=\alpha \ell(n-1) .
$$

The claim follows.

By combining Local Claims 1 and 2, we have $N L \leq L+\alpha \ell(n-1)$, from which the desired lower bound for $\alpha$ immediately follows.

\subsection{Lower Bound on the Number of Random Bits}

Let us now prove the lower bound on the number of random bits needed by the sender in a natural reduction.

Theorem 5. In any informationally-theoretic natural reduction of weak $\left(\begin{array}{c}N \\ 1\end{array}\right)$ $O T_{2}^{L}$ to $\left(\begin{array}{c}n \\ 1\end{array}\right)-O T_{2}^{\ell}$ the sender must flip at least $\frac{L(N-n)}{n-1}$ random coins.

Proof. Let $P, P=($ Alice, $B o b)$, be an information-theoretically secure natural reduction from weak $\left(\begin{array}{c}N \\ 1\end{array}\right)-\mathrm{OT}_{2}^{L}$ to $\left(\begin{array}{l}n \\ 1\end{array}\right)-\mathrm{OT}_{2}^{\ell}$. As before, let $W$ be the random input of Alice, $R$ be her random tape, $M_{s}$ be her ordinary messages sent to Bob and $V$ be her "potential" messages. We notice that since the reduction is natural, the distribution of $V$ and $M_{s}$ does not depend on Bob's index and his random string. Let $V_{j}, j=1 \ldots n$, be an $\alpha$-tuple consisting of string number $j$ taken from each of the $\alpha$ invocations of $\left(\begin{array}{l}n \\ 1\end{array}\right)-\mathrm{OT}_{2}^{\ell}$. We see that $V$ is the disjoint union of $V_{1}, \ldots, V_{n}$.

As before, we proceed by expanding the mutual information between $W$ and $\left(V, M_{s}\right)$ in two different ways.

$$
\mathbf{I}\left(\left(V, M_{s}\right) ; W\right)=\mathbf{H}(W)-\mathbf{H}\left(W \mid\left(V, M_{s}\right)\right)=N L-0=N L
$$

Here we used the fact that $W$ is determined from $V$ and $M_{s}$. Indeed, since $V$ and $M_{s}$ do not depend on Bob's input or random string, Alice should make sure that honest Bob can retrieve any $W_{i}$ with probability 1 (if his input is $i$ ).

On the other hand, it is a possible behavior for a (malicious) Bob to read string number $j$ in all the OT-rounds, i.e. to obtain $V_{j}$. By the weak sender

$\overline{2}$ Again, same result applies to (strong) $\left(\begin{array}{c}N \\ 1\end{array}\right)-\mathrm{OT}_{2}^{L}$ as well. 
privacy condition, $I\left(\left(V_{j}, M_{s}\right) ; W\right) \leq L$, and, therefore, for any $j \in[1, n]$ we have (using Lemma 2, equations 5 and 6)

$$
\mathbf{I}\left(\left(V, M_{s}\right) ; W\right)=\mathbf{I}\left(\left(V_{j}, M_{s}\right) ; W\right)+\mathbf{I}\left(V \backslash V_{j} ; W \mid\left(V_{j}, M_{s}\right)\right) \leq L+\mathbf{H}\left(V \backslash V_{j} \mid V_{j}\right)
$$

Combining this with Equation (5), we get

$$
\mathbf{H}\left(V \backslash V_{j} \mid V_{j}\right) \geq L(N-1), \quad \forall j \in[1, n]
$$

Since $V$ is a disjoint union of $V_{j}$ 's, we get from the above equation (for $j=n$ ) and Lemma2 (equations 1 and 5) that $L(N-1) \leq \mathbf{H}\left(V \backslash V_{n} \mid V_{n}\right) \leq \sum_{j=1}^{n-1} \mathbf{H}\left(V_{j} \mid V_{n}\right)$. Hence, there is an index $j \in[1, n-1]$ s.t. $\mathbf{H}\left(V_{j}\right) \geq \mathbf{H}\left(V_{j} \mid V_{n}\right) \geq \frac{L(N-1)}{n-1}$. W.l.o.g. assume $j=1$, i.e. $\mathbf{H}\left(V_{1}\right) \geq \frac{L(N-1)}{n-1}$. Since for a fixed $W$, the only randomness of $V$ came from $R$, we have by Equation (6) and Lemma 2 (equation 1)

$$
\begin{aligned}
|R| & \geq \mathbf{H}(V \mid W)=\mathbf{H}(V, W)-\mathbf{H}(W)=\mathbf{H}\left(V_{1}\right)+\mathbf{H}\left(V \backslash V_{1} \mid V_{1}\right)-N L \\
& \geq \frac{L(N-1)}{n-1}+L(N-1)-L N=\frac{L(N-n)}{n-1}
\end{aligned}
$$

Here $\mathbf{H}(V, W)=\mathbf{H}(V)$ as $W$ is a function of $V$, and then we use (6) for $j=1$ and our assumption on $\mathbf{H}\left(V_{1}\right)$. This completes the lower bound proof.

\section{Upper Bounds}

Though this paper focuses on proving lower bounds, we need to touch briefly upon upper bounds to demonstrate the tightness of Theorems 4 and 5 . This is done by means of a single natural reduction of weak $\left(\begin{array}{c}N \\ 1\end{array}\right)-\mathrm{OT}_{2}^{L}$ to $\left(\begin{array}{c}n \\ 1\end{array}\right)-\mathrm{OT}_{2}^{\ell}$ that simultaneously achieves both the lower bounds for the number of invocations of $\left(\begin{array}{l}n \\ 1\end{array}\right)-\mathrm{OT}_{2}^{\ell}$ and the number of random bits needed by the sender. This protocol is a simple generalization of the one given by Brassard, Crépeau and Sántha [5] for the case $L=\ell, n=2$. For completeness purposes, we also include the proof that this protocol works. Though a similar proof could be derived from [5], the one included here is more direct because our definition of a reduction is slightly simpler 3 Note that the same protocol also proves that our lower bounds are tight for reduction of (strong) $\left(\begin{array}{c}N \\ 1\end{array}\right)-\mathrm{OT}_{2}^{\ell}$ to $\left(\begin{array}{l}n \\ 1\end{array}\right)-\mathrm{OT}_{2}^{\ell}$.

Theorem 6. There exists a natural information-theoretically secure reduction of weak $\left(\begin{array}{c}N \\ 1\end{array}\right)-O T_{2}^{L}$ to $\left(\begin{array}{l}n \\ 1\end{array}\right)-O T_{2}^{\ell}$ such that

- it uses $\frac{L}{\ell} \cdot \frac{N-1}{n-1}$ invocations of $\left(\begin{array}{l}n \\ 1\end{array}\right)-O T_{2}^{\ell}$.

- the sender uses $\frac{L(N-n)}{n-1}$ random bits.

${ }^{3}$ You might notice, we embed the security of $\left(\begin{array}{l}n \\ 1\end{array}\right)-\mathrm{OT}_{2}^{\ell}$ into the definition of our reduction. Without doing so, one would have to argue about "nested mutual information". 
Moreover, for $L=\ell$, the reduction actually is a reduction of (strong) $\left(\begin{array}{c}N \\ 1\end{array}\right)-O T_{2}^{\ell}$ to $\left(\begin{array}{c}n \\ 1\end{array}\right)-O T_{2}^{\ell}$.

Proof. We start with $L=\ell$, i.e. a reduction of (strong) $\left(\begin{array}{c}N \\ 1\end{array}\right)-\mathrm{OT}_{2}^{\ell}$ to $\left(\begin{array}{c}n \\ 1\end{array}\right)-\mathrm{OT}_{2}^{\ell}$, making $\alpha=\frac{N-1}{n-1}$ invocations and using $\frac{\ell(N-n)}{n-1}$ random bits for Alice. Let $w=$ $w_{1}, \ldots, w_{N}$ be Alice's $N$ strings of length $\ell$ each, and let $i$ be Bob's index.

Protocol $P(w, i)$ :

1. Alice chooses $(\alpha-1)$ random $\ell$-bit strings $x_{1}, \ldots, x_{\alpha-1}$ using $\ell(\alpha-1)=$ $\frac{\ell(N-n)}{n-1}$ random bits. Set $x_{0}=0^{\ell}, x_{\alpha}=w_{N}$.

2. Perform $\alpha$ invocations of the $\left(\begin{array}{l}n \\ 1\end{array}\right)-\mathrm{OT}_{2}^{\ell}$ where transfer $j=0 \ldots(\alpha-1)$ implements

$\left(\begin{array}{c}n \\ 1\end{array}\right)-\mathrm{OT}_{2}^{\ell}\left[w_{j(n-1)+1} \oplus x_{j}, w_{j(n-1)+2} \oplus x_{j}, \ldots, w_{(j+1)(n-1)} \oplus x_{j}, x_{j+1} \oplus x_{j}\right]$.

Let $z_{j}$ be the value Bob reads from the $j^{\text {th }}$ invocation, described next.

3. Let $j_{0} \in\{0 \ldots(\alpha-1)\}$ be the index of the box which has the XOR-ed value of $w_{i}\left(=\left\lfloor\frac{i-1}{n-1}\right\rfloor\right.$, if $i \neq N$, and $=(\alpha-1)$, otherwise $)$. Bob reads the value $z_{j_{0}}=w_{i} \oplus x_{j_{0}}$ from box number $j_{0}$ and values $z_{j}=x_{j+1} \oplus x_{j}$ for all $j \neq j_{0}$.

4. Bob outputs $\bigoplus_{j=0}^{j_{0}} z_{j}$.

We now prove that the above protocol indeed implements strong $\left(\begin{array}{c}N \\ 1\end{array}\right)-\mathrm{OT}_{2}^{\ell}$. The Correctness Property $(\mathrm{P} 1)$ is clear since $\left(w_{i} \oplus x_{j_{0}}\right) \oplus\left(x_{j_{0}} \oplus x_{j_{0}-1}\right) \oplus \ldots\left(x_{2} \oplus\right.$ $\left.x_{1}\right) \oplus x_{1}=w_{i}$. The Receiver Privacy (P2) is clear as well since the scheme is natural and, as we just saw, Bob can recover any $w_{i}$. We now show the main condition (P3).

Let $W=W_{1}, \ldots, W_{N}$ be chosen at random as well as Alice's random string $R=X_{1}, \ldots, X_{\alpha-1}$. Let $V$ be the random variable containing all $(\alpha n)$ values of the $\left(\begin{array}{l}n \\ 1\end{array}\right)-\mathrm{OT}_{2}^{\ell}$ boxes. We can assume w.l.o.g. that in each of the $\alpha$ OT boxes, Bob indeed read one entire $\ell$-bit string that he chose (he can not learn more and it "does not hurt" to learn as much as possible). Thus, define $V_{r}$ to be the $\alpha$-tuple of $\ell$-bit strings that Bob read, i.e. everything that Bob learned from the protocol. Let $t_{0}, \ldots, t_{\alpha-1}$, where $t_{j} \in[1, n]$, be the (random variables denoting the) indices of $\alpha$ strings that Bob read.

Let $j_{0}$ be the smallest number such that $t_{j_{0}} \neq n$, if it exists. Otherwise, $j_{0}=$ $\alpha-1$. Thus, Bob learned $X_{1}, X_{1} \oplus X_{2}, \ldots, X_{j_{0}-2} \oplus X_{j_{0}-1}$ and some $W_{i} \oplus X_{j_{0}-1}$. Clearly, this enables him to reconstruct $W_{i}$ (the exceptional case of all $t_{j}=n$ falls here as well giving Bob $\left.W_{N}\right)$. We let $\tilde{I}=i$. First of all, $\tilde{I}$ is independent from $W$. Indeed, Bob choose to read index $t_{j_{0}}$ in the $j_{0}^{t h}$ invocation of $\left(\begin{array}{l}n \\ 1\end{array}\right)$-OT $\mathrm{OT}_{2}^{\ell}$ only based on his random coins and $X_{1}, X_{1} \oplus X_{2}, \ldots, X_{j_{0}-2} \oplus X_{j_{0}-1}$, which does not depend on $W$. Thus, it suffices to show that $\mathbf{I}\left(V_{r} ; W \mid W_{\tilde{I}}\right)=0$. But we already observed that $W_{\tilde{I}}$ is determined from $V_{r}$. Hence, using Lemma 2 (equations 4 and 3),

$$
\begin{aligned}
\mathbf{I}\left(V_{r} ; W\right) & =\mathbf{I}\left(\left(V_{r}, W_{\tilde{I}}\right) ; W\right)=\mathbf{I}\left(W_{\tilde{I}} ; W\right)+\mathbf{I}\left(V_{r} ; W \mid W_{\tilde{I}}\right) \\
& =\ell+\mathbf{I}\left(V_{r} ; W \mid W_{\tilde{I}}\right)
\end{aligned}
$$


Thus, we only need to show that $\mathbf{I}\left(V_{r} ; W\right)=\ell$, i.e. to establish the weak property $\left(\mathrm{P} 3^{\prime}\right)$. Intuitively, Bob always learns some $W_{\tilde{I}}$, i.e. $\ell$ bits of information. So if we show that he does not learn more than $\ell$ bits of information, we know that the only thing he learned was that one string $W_{\tilde{I}}$. We proceed by showing a sequence of easy claims.

Local Claim 1: $W$ is a function of $V$, i.e.

$$
\mathbf{H}(W \mid V)=0
$$

Proof: We already saw from correctness that $V$ determines each string $W_{i}$.

Local Claim 2:

$$
\mathbf{H}\left(V \backslash V_{r} \mid V_{r}\right)=\ell(N-1)
$$

Proof: We show that all $(\alpha n)$-bit strings of $V$ are totally independent when $W$ and $R$ are randomly chosen. Let us view each such string in $V$ as an $(N+$ $\alpha-1)$-dimensional vector over $\mathbb{Z}_{2}$ by taking the characteristic vector of the equation defining this string. Since all $W_{i}$ and $X_{j}$ are chosen randomly, our strings are independent iff the corresponding vectors are linearly independent. Assume that some linear combination of vectors in $V$ is zero. This combination cannot include a vector depending on some $W_{i}$ as there is only one such vector in $V$. And the remaining vectors $X_{1}, X_{1} \oplus X_{2}, \ldots, X_{\alpha-2} \oplus X_{\alpha-1}$ are clearly linearly independent. And since our disjoint split of $V$ into $V_{r}$ and $V \backslash V_{r}$ does not depend on $V \backslash V_{r}$, we get that $V \backslash V_{r}$ is independent of $V_{r}$, so by Lemma 2 (equation 5), $\mathbf{H}\left(V \backslash V_{r} \mid V_{r}\right)=\left|V \backslash V_{r}\right|=\ell(n-1) \alpha=\ell(N-1)$.

Local Claim 3: $V \backslash V_{r}$ is determined from $W$ and $V_{r}$, i.e.

$$
\mathbf{H}\left(V \backslash V_{r} \mid\left(V_{r}, W\right)\right)=0
$$

Proof: The knowledge of $W$ and any string $W_{i} \oplus X_{\alpha-1}$ in the last $\left(\begin{array}{l}n \\ 1\end{array}\right)$-OT $T_{2}^{\ell}$ box (which we have from $V_{r}$ ) determines $X_{\alpha-1}$. Knowing $X_{\alpha-1}, W$ and any string of the form $z \oplus X_{\alpha-2}$ from the next to last $\left(\begin{array}{l}n \\ 1\end{array}\right)$-OT $\mathrm{OT}_{2}^{\ell}$ box (which we have from $V_{r}$ where $z$ is either some $W_{i}$ or $X_{\alpha-1}$ ) enables one to deduce $X_{\alpha-2}$. Continuing this way, we determine $X_{1}$ from the first $\left(\begin{array}{c}n \\ 1\end{array}\right)-\mathrm{OT}_{2}^{\ell}$ box which allows us to reconstruct the whole $V \backslash V_{r}$.

Combining Local Claims 1,2,3 and using Lemma 2 (equations 1, 2 and 3),

$$
\begin{aligned}
\ell N & =\mathbf{H}(W)=\mathbf{H}(W)-\mathbf{H}(W \mid V)=\mathbf{I}(V ; W)=\mathbf{I}\left(V_{r} ; W\right)+\mathbf{I}\left(V \backslash V_{r} ; W \mid V_{r}\right) \\
& =\mathbf{I}\left(V_{r} ; W\right)+\mathbf{H}\left(V \backslash V_{r} \mid V_{r}\right)-\mathbf{H}\left(V \backslash V_{r} \mid\left(V_{r}, W\right)\right)=\mathbf{I}\left(V_{r} ; W\right)+\ell(N-1)
\end{aligned}
$$

Hence, $\mathbf{I}\left(V_{r} ; W\right)=\ell$ indeed. This completes the proof of correctness when $L=\ell$.

For $\ell<L$ we give a trivial protocol that sacrifices the strong property (P3) leaving only $\left(\mathrm{P} 3^{\prime}\right)$. The protocol simply splits each of the strings of the database 
into $L / \ell$ disjoint parts of length $\ell$ each, and performs the previous protocol implementing $\left(\begin{array}{c}N \\ 1\end{array}\right)-\mathrm{OT}_{2}^{\ell}$ using $\left(\begin{array}{c}n \\ 1\end{array}\right)-\mathrm{OT}_{2}^{\ell}$. It uses $\frac{L}{\ell} \cdot \frac{N-1}{n-1}$ invocations of $\left(\begin{array}{c}n \\ 1\end{array}\right)-\mathrm{OT}_{2}^{\ell}$ and $\frac{L}{\ell} \cdot \frac{\ell(N-n)}{n-1}=\frac{L(N-n)}{n-1}$ random bits as claimed. The correctness is clear except Alice's privacy. We clearly loose the strong property (P3) as Bob can learn up to $L / \ell$ different blocks of length $\ell$ from different strings. However, weak property $\left(\mathrm{P} 3^{\prime}\right)$ still holds as the $L / \ell$ groups of boxes are totally independent, and from each of them Bob can learn at most $\ell$ bits about $W$, i.e. a total of at most $\ell \cdot \frac{L}{\ell}=L$ bits.

\section{Acknowledgments}

We would like to thank Amos Lapidoth for his critical comments about this paper. Special thanks to Madhu Sudan and Peter Winkler for useful remarks and suggestions.

\section{References}

1. M. Bellare, S. Micali. Non-interactive Oblivious Transfer and Applications. In Advances in Cryptology: Proceedings of Crypto '90, pp. 547-559, Springer-Verlag, 1990.

2. M. Blum. How to Exchange (Secret) Keys. In ACM Transactions of Computer Systems, vol. 1, No. 2, pp. 175-193, May 1983.

3. G. Brassard, C. Crépeau. Oblivious Transfers and Privacy Amplification. In Advances in Cryptology: Proceedings of Eurocrypt '97, Springer-Verlag, pp. 334347, 1997.

4. G. Brassard, C. Crépeau, J. Robert. Information theoretic reductions among disclosure problems. In 27th Symp. of Found. of Computer Sci., pp. 168-173, IEEE, 1986.

5. G. Brassard, C. Crépeau, M. Sántha. Oblivious Transfers and Intersecting Codes. In IEEE Transaction on Information Theory, special issue in coding and complexity, Volume 42, Number 6, pp. 1769-1780, 1996.

6. C. Crépeau. Equivalence between two flavors of oblivious transfers. In $A d$ vances in Cryptology: Proceedings of Crypto '87, volume 293 of Lecture Notes in Computer Science, pp. 350-354, Springer-Verlag, 1988.

7. C. Crépeau. A zero-knowledge poker protocol that achieves confidentiality of the players' strategy or how to achieve an electronic poker face. In Advances in Cryptology: Proceedings of Crypto '86, pp. 239-247. Springer-Verlag, 1987.

8. C. Crépeau, J. Kilian. Weakening security assumptions and oblivious transfer. In Advances in Cryptology: Proceedings of Crypto '88, volume 403 of Lecture Notes in Computer Science, pp. 2-7, Springer-Verlag, 1990.

9. S. Even, O. Goldreich, A. Lempel. A Randomized Protocol for Signing Contracts. In Advances of Cryptology: Proceedings of Crypto '83, Plenum Press, New York, pp. 205-210, 1983.

10. M. Fisher, S. Micali, C. Rackoff. A Secure Protocol for the Oblivious Transfer. In Journal of Cryptology, vol. 9, No. 3 pp. 191-195, 1996.

11. O. Goldreich, S. Micali, A. Wigderson. How to play any mental game, or: A completeness theorem for protocols with honest majority. In Proceedings of 19th Annual Symp. on Theory of Computing, 218-229, 1987. 
12. S. Goldwasser, S. Micali, C. Rackoff. The knowledge complexity of interactive proof-systems. In SIAM Journal on Computing, 18:186-208, 1989.

13. J. Kilian. Founding Cryptography on Oblivious Transfer. In Proceedings of 20th Annual Symp. on Theory of Computing, pp. 20-31, 1988.

14. J. Kilian, S. Micali, R. Ostrovsky. Minimum Resource Zero-Knowledge Proofs. In Proceedings of 30th Annual Symp. on Foundations of Computer Science, pp. 474-479, 1989.

15. S. Micali, P. Rogaway. Secure Computation. In Advances in Cryptology: Crypto' 91 Proceedings, pp. 392-404, Springer-Verlag, 1992.

16. H. Nurmi, A. Salomaa, L. Santean. Secret ballot elections in computer networks. In Computer and Security, volume 10, No. 6, pp. 553-560, 1991.

17. M. Rabin. How to Exchange Secrets by Oblivious Transfer. In Technical Memo TR-81, Aiken Computation Laboratory, Harvard University, 1981. 